\title{
Revisión sistemática: Evaluación de la exactitud y precisión en el diagnóstico de depresión
}

Is this patient clinically depressed? Williams JW, Noël PH, Cordes JA et al. JAMA 2002; 287:1160-1170.

\section{Objetivo}

Revisar la exactitud y precisión de los instrumentos de búsqueda de casos (cuestionarios) y de la evaluación clínica en el diagnóstico de depresión

\section{Fuente de Datos}

Búsqueda de MEDLINE y de una base de datos de ensayos clínicos de Cochrane. Limitada a la literatura de habla inglesa desde 1970 a Julio 2000 . No se buscaron datos no publicados.

\section{Selección de Datos}

Se incluyeron estudios realizados en el ámbito de la atención primaria que evaluaban a por lo menos 100 pacientes, y que utilizaban un cuestionario fácil de entender y de calcular así como rápido de realizar (1 a 5 min.).Se requirió que hubiera una comparación con un diagnóstico basado en un criterio estandarizado de depresión como test de referencia o gold standard* (ej, entrevistas psiquiátricas con criterios del DSM).Se incluyeron 28 estudios, que utilizaban 11 instrumentos diferentes.

Dentro de los estudios de evaluación clínica se incluyeron aquellos que consistían en la evaluación de por lo menos dos profesionales y que realizaban un diagnóstico de depresión basado en criterios estandarizados.Se incluyeron tanto entrevistas semiestructuradas como no estructuradas.No se seleccionó según tipo especial de población. Se incluyeron 14 estudios y todos incluían profesionales especializados en salud mental.

\section{Extracción de datos}

Los datos fueron analizados y sintetizados por dos revisores independientes. En los estudios sobre cuestionarios se evaluó la calidad según:tamaño de muestra, aleatorización, enmascaramiento y proporción de pacientes que fueron evaluados para realizar el diagnóstico según el criterio patrón establecido.Según este criterio 15 de los 28 estudios ( $54 \%$ del total) eran de una razonable calidad para la evaluación de los datos.

En los estudios sobre evaluación clínica se tomó en cuenta si había: descripción de la severidad del cuadro, independencia en la recolección de la historia clínica, y enmascaramiento. La calidad de estos estudios fue muy variable.

Los datos de los cuestionarios fueron combinados cuantitativamente y se calcularon los coeficientes de probabilidad positiva y negativa*. Los estudios de entrevista no fueron combinados cuantitativamente debido a una marcada heterogeneidad de los estudios.

\section{Resultados Principales}

Los datos obtenidos de los estudios con cuestionarios tuvieron una mediana de los coeficientes de probabilidad positiva de 3,3 (IC95\% $2,3$ a 12,2$)$ y una mediana de los coeficientes de probabilidad negativa de 0,19 (IC95\% 0,14 a 0,35). No se encontró diferencias significativas entre los cuestionarios.

Se identificaron siete estudios semiestructurados con trabajadores de salud mental.Se obtuvo un kappa ${ }^{*}=0,64-0.93$. Otros siete estudios no estructurados tuvieron un $\mathrm{k}=0,55-0,74$. Un estudio con médicos generalistas y psiquiatras demostró un $\mathrm{k}=0,71$ entre ambos grupos (buen grado de acuerdo)

\section{Conclusiones}

Existen varios cuestionarios para detectar depresión que son eficaces. El diagnóstico confirmatorio clínico de los profesionales de la salud mental es altamente confiable.Un estudio demostró una alta confiabilidad en el diagnóstico realizado por los médicos generalistas.

Fuente de financiamiento:No referida

\section{Comentario}

El presente artículo pertenece a la serie The Rational Clinical Examination que se publica en JAMA desde hace aproximadamente 10 años. ${ }^{1}$ Es parte de un esfuerzo colaborativo para promover el estudio analítico de la evaluación clínica, incluyendo tanto el interrogatorio como el examen físico. ${ }^{2}$

Los trastornos depresivos son altamente prevalentes, pero el diagnóstico suele ser difícil, en parte debido a que es un diagnóstico clínico, sindromático.En repetidas ocasiones se ha documentado que los médicos de atención primaria no reconocen la depresión mayor en aproximadamente la mitad de los pacientes que la padecen.

Habría dos estrategias para detectar y diagnosticar pacientes con depresión.Una es la "búsqueda de caso", en la cual rutinariamente a todos los pacientes que concurren a una consulta cualquiera se los invita a completar un cuestionario.Aquellos que obtienen un puntaje por encima de un umbral presentan mayor chance de presentar depresión y por lo tanto son evaluados clínicamente para depresión. Una segunda estrategia consiste en evaluar sólo a aquellos pacientes que inspiran una sospecha clínica, por ejemplo aquellos con enfermedades crónicas, con síndromes dolorosos crónicos, o con síntomas físicos múltiples y no explicados.

Existen múltiples instrumentos tipo cuestionario para la detección de pacientes deprimidos para el uso rutinario en atención primaria. ${ }^{3}$ Entre estos, los siguientes fueron diseñados específicamente para detectar depresión y son los más evaluados en atención primaria: Beck Depresión Inventory (BDI), Center for Epimiologic Studies Depresión Screen (CES-D), y Zung Self-Assesment Depresión Scale (SDS). ${ }^{46}$ Estos instrumentos permiten determinar la severidad del cuadro y por lo tanto son útiles para monitorear la respuesta al tratamiento.Algunos de ellos fueron validados en español (por ejemplo SDS7 o CES-D8), y alguno como el Beck en Argentina. ${ }^{9}$

En el año 2002 se actualizaron las recomendaciones de Las Fuerzas de Tareas Americanas y actualmente dan una recomendación de tipo $\mathrm{B}^{*}$ para el rastreo de depresión. ${ }^{10}$

Conclusiones del comentador: la depresión es un cuadro prevalente de alta morbilidad, muchas veces no diagnosticado a pesar de ser detectable eficazmente a través de instrumentos disponibles en atención primaria. Es importante tener en cuenta que el uso de estas estrategias genera más diagnósticos y que hay que estar preparado para abordar los casos detectados en forma adecuada.

Juan L. Bernabó [ Servicio de Clínica Médica.Hospital Italiano de Buenos Aires. ]

*Ver glosar io

Referencias:

1. Sackett DL, Rennie D.The science of the art of the clinical examination..JAMA 1992.267(19):2650-2

2. http://www.sgim.org/clinexam.cfm

3. Mulrow CD, Williams JW, Gerety MB et al.Case-findings instruments for depression in primary care setting.Ann Intern Med.1995;12:913-921

4. BDI:http://www.uea.ac.uk/ wp316/depression.pdf

5. CES-D:http://chipts.ucla.edu/pdf/assessments/Depression CES-D.pdf

6. SDS:http://www.wellbutrin-sr.com/hcp/graphics/zung.pdf

7. Aragones Benaiges E, Masdeu Montala RM, Cando Guasch G, Coll Borras G.Diagnostic validity of Zung's self-rating depression scale on primary care patients.Actas Esp Psiquiatr. 2001 Sep-Oct;29(5):310-6

8. Bonicatto S, Dew AM, Soria JJ. Analysis of the psychometric properties of the Spanish version of the Beck Depression Inventory in Argentina.Psychiatry Res. 1998 Jul 13;79(3):277-85.

9. Soler J, Perez-Sola V, Puigdemont D, Perez-Blanco J, Figueres M, Alvarez E. Validation study of the Center for Epidemiological Studies-Depression of a Spanish population of patients with affective disorders.Actas Luso Esp Neurol Psiquiatr Cienc Afines.1997 Jul-Aug;25(4):243-9.

10. U.S.Preventive Services Task Force.Screening for depression:recommendations and rationale.Ann Intern Med.136(10):760-64 